\title{
Antibiofilm coatings based on protein-engineered polymers and antimicrobial peptides for preventing implant-associated infections
}

Sergio Acosta, Arturo Ibañez-Fonseca, Conrado Aparicio* and J. Carlos RodríguezCabello*

Dr. Sergio Acosta, Dr. Arturo Ibañez-Fonseca and Prof. Dr. J. Carlos RodríguezCabello

Bioforge lab, CIBER-BBN, Edificio LUCIA, University of Valladolid, Paseo Belén 19, Valladolid 47011, Spain

Prof. Dr. Conrado Aparicio

MDRCBB, Minnesota Dental Research Center for Biomaterials and Biomechanics, University of Minnesota, 16-250A Moos Tower, 515 Delaware Street Southeast, Minneapolis, Minnesota 55455, United States

*apari003@umn.edu

*roca@bioforge.uva.es

Keywords: antibiofilm, coatings, protein engineering, antimicrobial peptides, implant infections

\begin{abstract}
Implant-associated infections (IAIs) are one of the leading concerns in orthopedics and dentistry as they commonly lead to implant failure. The presence of biofilms and, increasingly frequently, drug-resistant bacteria further impairs the efficacy of conventional antibiotics. Immobilization of antimicrobial peptides (AMPs) on implant surface is a promising alternative to antibiotics for prevention of IAIs. In addition, the use of functional linkers for the AMP tethering enables to increase the antimicrobial potential and the bioactivities of the coating. In this study, an extracellular-matrix-mimicking system based on elastin-like recombinamers (ELRs) has been developed for the covalent anchoring of AMPs and investigated for use as a hybrid antibiofilm coating. A drip-flow biofilm reactor was used to simulate in vivo environmental dynamic conditions, thus showing that the presence of the AMPs in the hybrid coatings provided strong antibiofilm
\end{abstract}


activity against monospecies and microcosm biofilm models of clinical relevance. These results, together with an excellent cytocompatibility towards primary gingival fibroblasts, encourage the use of ELRs as multivalent platforms for AMPs and open up a wide range of possibilities in the biofabrication of advanced coatings combining the antibiofilm potential of AMPs and the outstanding tunability and biomechanical properties of the ELRs. 


\section{Introduction}

Implantable biomedical devices have revolutionized patient rehabilitation, although their use increases the risk of infection. The implantation of a medical device provides an opportunity for nosocomial pathogens to colonize the site and form a biofilm on the device surface, thus leading to infections that may cause prolonged hospitalizations and higher mortality rates. ${ }^{1}$ In fact, implant-associated infections (IAIs) are one of the most common and severe complications of healthcare interventions, ${ }^{2,3}$ thereby creating an immense economic and social burden. ${ }^{4}$ Furthermore, in many cases conventional antibiotics are ineffective at treating these types of infections due to the presence of biofilms and the increasing prevalence of antibiotic-resistant bacteria. ${ }^{5}$ Thus, the development of new strategies to prevent device colonization and biofilm formation is essential.

Antimicrobial peptides (AMPs) and designer AMPs are some of the most promising alternatives to conventional antibiotics. AMPs are anti-infective agents that are present in animals, plants and bacteria. Most of them are small (10-50 amino acids) and cationic peptides with amphipathic behavior that enables them to interact with multiple biomolecules. As such, AMPs exhibit a broad spectrum of activity against Gram positive and Gram negative bacteria, fungi and viruses as well as the ability to modulate the host's innate immunity. ${ }^{6}$ However, the molecular mechanism of action of most AMPs is not entirely understood. In solution, AMPs are able to interpenetrate the bacterial outer membrane or the cell wall, interacting with the cell membrane and intracellular targets and killing bacteria by inducing membrane permeability or inhibiting intracellular processes. ${ }^{7}$ However, they may also show toxicity and sensitivity to proteases that limits their application. ${ }^{8}$ Notably, immobilization of AMPs onto surfaces enhances their stability and increases their local concentration and biological availability but it 
compromises their ability to interact with multiple targets. ${ }^{9}$ Although AMPs may show bactericidal and antibiofilm activity when directly immobilized onto surfaces, ${ }^{10-14}$ recent studies suggest that attachment of the peptides to the substrate via a spacer enhances the flexibility, exposure and functional conformation of the AMPs, thereby improving the anti-infective potential of the coatings. ${ }^{15-19}$ This approach can also be used to produce multivalent coatings with improved features for clinical use, such as anti-adhesive properties, high affinity for biomaterial, or biomimetic behavior to enhance tissue integration of permanent orthopedic and dental implants. ${ }^{13,20,21}$ As such, the strategy used to immobilize AMPs plays a key role in the biological response of the functional coating in terms of biocompatibility and antimicrobial properties. In this sense, extracellular matrix (ECM)-inspired polymers, such as elastin-like recombinamers (ELRs), are attractive candidates for the fabrication of bioactive coatings that incorporate AMPs on implant surface.

ELRs are protein-engineered polymers inspired by intrinsically disordered domains of the tropoelastin, that mimic its unique biomechanical properties. ${ }^{22}$ They are biocompatible, non-immunogenic, ${ }^{23}$ and their recombinant nature offers both tunable bioactivity and extreme control over composition, properties that are lacking in many synthetic and natural polymers. ${ }^{24}$ Indeed, ELRs have demonstrated their applicability as covalent coatings, offering a versatile coating to control the biological response of biomedical materials, improving tissue integration and biocompatibility of the device, or incorporating additional functionalities that include control of biomineralization or spatial control over cell adhesion to produce cellular micropatterns. ${ }^{25-28}$

In the present study, we have developed an ECM-mimicking system based on ELRs to tether AMPs on indwelling devices and we have assessed their potential use as a cytocompatible and antibiofilm coating for preventing implant infections. The versatility 
of the biomimetic platform allowed both enantiomeric forms ( $\mathrm{L}$ and $\mathrm{D})$ of the designer peptide GL13K (GKIIKLKASLKLL-NH ${ }_{2}$ ) to be incorporated into the polymeric backbone by genetic engineering or chemical derivatization, respectively. The D- and Lenantiomers of GL13K peptide have been shown to have different self-assembly properties that can predetermine their antimicrobial activity in solution. ${ }^{29-31}$ Overall, the D-enantiomer of GL13K is a protease resistant peptide that can evade bacterial resistance. ${ }^{32}$ The resulting hybrid ELRs were subsequently tethered to commercially pure titanium discs and their biological response was tested. Cytocompatibility was assessed against human primary cells and their antibiofilm efficacy against two oral biofilm models of clinical relevance. In order to assess the response of the coatings against the early colonizers of the oral cavity, Streptococcus gordonii was tested since it initiates dental plaque formation and facilitates the attachment of secondary pathogenic colonizers, such as Porphyromonas gingivalis. ${ }^{33}$ An oral microcosm biofilm model derived from cariogenic dental plaque was also assayed in order to mimic the high complexity and heterogeneity of the oral microbiota. ${ }^{34}$ Both oral biofilm models were evaluated under dynamic conditions using a drip flow biofilm reactor (DFBR). The DFBR enables in vitro simulation of some of the relevant conditions for bacteria biofilm development in vivo. A constant drip flow is provided during the whole incubation, thus mimicking the continuous nutritional supply and shear forces found in the oral cavity. Therefore, the antibiofilm properties of the coatings were tested under more challenging and realistic conditions than when using conventional static culture methods. ${ }^{11}$ The goal of this study was to evaluate the antibiofilm potential and cytocompatibility of the resulting hybrid coatings in order to determine the applicability of ELRs as multivalent platforms for AMP coatings. 


\section{Materials and methods}

\subsection{AMP/ELR/AMP-ELR synthesis}

All the ELRs in this work (Table 1) were based on the polycationic recombinamer VC. VC, used as a negative control, and the hybrid ELR, which contained the L-enantiomer of GL13K (VCL), were recombinantly produced in E. coli BLR (DE3) as described elsewhere. ${ }^{35}$ For production of the hybrid ELR containing D-enantiomers of the GL13K (VCD), the lysine side chains of VC were chemically modified using 'click chemistry', namely strain-promoted azide-alkyne cycloaddition (SPAAC), with the designer Dpeptide, following the method previously reported by Gonzalez de Torre et al. and represented schematically in Figure $1 .^{36}$

First, approximately four amine groups on VC were modified with cyclooctyne groups (Figure S1, S2 and Table S1), then an average of three D-GL13K peptides were linked to each VC-cyclooctyne molecule via a SPAAC reaction (Figure S3 and Table S1). DGL13K functionalized with an azide group at its $\mathrm{N}$-terminus $\left(\mathrm{N}_{3}\right.$-Gkiiklkaslkll- $\left.\mathrm{NH}_{2}\right)$ was purchased from Pepscan (the Netherlands) with a purity $>90 \%$ (Figure S4). D-GL13K peptide (Gkiiklkaslkll-NH ), used as a positive control, was synthesized by AAPPTec, LLC (USA) with a purity $>99 \%$ (Figure S5).

\subsection{Covalent functionalization of titanium surfaces}

Immobilization of the AMP/ELR/AMP-ELRs onto titanium surfaces was performed using organosilanes as covalent linkers. As shown in Figure 2, Ti discs (six mm diameter) were fabricated from commercially pure (C.P.) titanium grade II sheets (McMaster-Carr ${ }^{\mathbb{R}}$, US). These discs were polished and alkaline etched (16 h, $\left.5 \mathrm{M} \mathrm{NaOH}, 60{ }^{\circ} \mathrm{C}\right)$, then the etched $\mathrm{Ti}(\mathrm{eTi})$ discs were washed with distilled water for $30 \mathrm{~min}$ (3 times) and modified 
with two different organosilanes, depending on the molecule to be immobilized. (3Chloropropyl)triethoxysilane (CPTES) was used to tether D-GL13K peptides via their amine functional groups, as described elsewhere. ${ }^{10}$ ELRs were tethered in an oriented manner via their C-terminal Cys-Cys motif using 3-aminopropyltriethoxysilane (APTES) and the bifunctional linker sulfosuccinimidyl 4-( $N$-maleimidomethyl) cyclohexane-1carboxylate (sulfo-SMCC). Thus, eTi discs were immersed in an anhydrous ethanol solution of APTES $(5 \%)$ at $60^{\circ} \mathrm{C}$ for $2 \mathrm{~h}$. After that, samples were rinsed and ultrasonicated in ethanol, distilled water, isopropanol and acetone (to remove physisorbed molecules), then dried under $\mathrm{N}_{2}$. Sulfo-SMCC was utilized to introduce the maleimide groups. Thus, APTES modified discs were immersed in a $1 \mathrm{mg} \mathrm{mL}^{-1}$ sulfo-SMCC solution (dissolved in sodium phosphate buffer $(\mathrm{PB}, \mathrm{pH}$ 7.2)) at room temperature (RT) for $1 \mathrm{~h}$, then rinsed and ultrasonicated in PB. Finally, the maleimide-modified Ti discs (SMCC) were immersed in ELR solutions $(0.2 \mathrm{mM}$ in $\mathrm{PB}, \mathrm{pH} 7.2)$ at $4{ }^{\circ} \mathrm{C}$ for $16 \mathrm{~h}$. Samples were subsequently washed with ultrapure water in triplicate, dried under $\mathrm{N}_{2}$ gas, sterilized with UV light (30 min per side) and stored at $-80^{\circ} \mathrm{C}$ until further use.

\subsection{Physicochemical characterization of the coatings}

Ti discs functionalized with AMPs, ELRs or AMP-ELRs and discs obtained from intermediate modification steps, were characterized to assess the effectiveness of the modification by evaluating the wettability of the surfaces measuring the water contact angle (WCA), and by quantification of the elemental composition using X-ray Photoelectron Spectroscopy (XPS), as described below.

\subsubsection{Water contact angle}

Dynamic water contact angle measurements were determined using the sessile drop technique and a DM-CE1 instrument (Kyowa Interface Science, Japan). At least six 2- $\mu \mathrm{L}$ 
drops of ultrapure water were analyzed. All images were collected at $100 \mathrm{~ms}$ after deposition of the drop and every second for 100 seconds. The left and right angles for each drop were averaged.

\subsection{2. $\quad X$-ray photoelectron spectroscopy}

A PHI 5000 VersaProbe III (ULVAC, Inc., Japan) X-ray photoelectron spectrometer equipped with a monochromatic $\mathrm{Al} \mathrm{K} \alpha \mathrm{X}$-ray source $\left(45^{\circ}, 1486.6 \mathrm{eV}, 50 \mathrm{~W}\right.$, sampling area; $200-\mu m$ diameter) was used to measure elemental composition. Survey spectra were collected using a pass energy of $280 \mathrm{eV}$ and a step size of $1.0 \mathrm{eV}$. At least five different samples were analyzed per group.

\subsection{Mechanical and thermal stability of the coatings}

Covalently coated $\mathrm{Ti}$ discs were ultrasonicated for $2 \mathrm{~h}$ in ultrapure water to test their mechanical stability in aqueous solvent. These discs were then incubated in phosphate buffered saline solution (PBS, pH 7.4) at $37^{\circ} \mathrm{C}$ for 2 weeks. The stability of the coatings was checked by XPS and WCA after ultrasonication for $2 \mathrm{~h}$ and after incubation for one week and two weeks in PBS at $37^{\circ} \mathrm{C}$.

\subsection{Oral biofilms culture}

Monospecies biofilms (S. gordonii DL-1) were grown on Ti discs coated with the protein polymers and the GL13K peptide; eTi discs were used as negative control. Biofilms were formed following a two stage method: 1) Inoculation/static stage: coated Ti discs and controls were inoculated by adding $2 \mathrm{~mL}$ of a $10^{7} \mathrm{CFU} \mathrm{mL} \mathrm{m}^{-1}$ bacterial inoculum from an overnight culture in Bacto Todd-Hewitt broth (THB; BD Biosciences, USA) at $37{ }^{\circ} \mathrm{C}$. Incubation during this stage was performed under static conditions in a 24-well plate for $4 \mathrm{~h}$ at $37^{\circ} \mathrm{C}$; 2) Growth/dynamic stage: discs were then transferred to a drip flow biofilm 
reactor (DFBR) (Biosurface Technologies Corp., USA) and incubated for $48 \mathrm{~h}$ at $37^{\circ} \mathrm{C}$ with a constant drip flow of $0.3 \mathrm{~mL} \mathrm{~min}{ }^{-1}$ of THB medium. In order to mimic the oral cavity conditions that the implant coatings are likely to experience, the drip flow rate was selected from the low end of the unstimulated salivary flow rate, $0.1-2 \mathrm{~mL} \mathrm{~min}^{-1} .{ }^{37}$ The salivary flow rate at the gingival sulcus, where the implant would be located, is expected to be much lower than in the open areas in the oral cavity. At the end of the experiment, Ti discs were carefully rinsed with $0.9 \% \mathrm{NaCl}$ solution to remove unattached and loosely attached bacteria.

Oral microcosm biofilms were produced using the 769-NS model collected and characterized by Rudney et al. ${ }^{38}$ At least 103 different taxons were identified within this biofilm model, which was produced from samples of whole saliva and plaque from a child at high risk of caries. Saliva samples were collected directly from the researcher who performed the experiments (healthy male, aged between 26 and 30 years, no periodontal disease) following established protocols, clarified by centrifugation and filtered through a $0.2-\mu \mathrm{m}$ filter. ${ }^{39} \mathrm{~A}$ two-stage method was again used for biofilm formation: 1) Inoculation/static stage: coated Ti discs and controls were first pre-incubated in filtered saliva for $10 \mathrm{~min}$ at RT in a 48 -well plate. Then, $10 \mu \mathrm{L}$ of $769-\mathrm{NS}$ glycerol stock was added to $20 \mathrm{~mL}$ of basal mucin medium (BMM), which is a complex medium used for the study of oral microcosm models, ${ }^{34,38,40}$ and the discs were inoculated under static conditions at $37{ }^{\circ} \mathrm{C}$ for 24 h. 2) Growth/dynamic stage: discs were transferred to the DFBR and incubated at $37{ }^{\circ} \mathrm{C}$ for 6 days with a drip flow of $0.3 \mathrm{~mL} \mathrm{~min}{ }^{-1}$ of BMM. Finally, the discs were gently rinsed with $0.9 \% \mathrm{NaCl}$ solution in a similar manner to the one-strain biofilm model. 


\subsection{Antibiofilm potency: CFU, ATP and DNA quantification}

For quantification of the antibiofilm potency of the coatings, the biofilms formed on the Ti discs were collected by ultrasonication in 330 or $660 \mu \mathrm{L}$ of $0.9 \% \mathrm{NaCl}$ solution, at 4

${ }^{\circ} \mathrm{C}$ for monospecies $S$. gordonii or microcosm biofilms, respectively. Subsequently, 100 $\mu \mathrm{L}$ of the bacterial extract was incubated for 2 hours at $37^{\circ} \mathrm{C}$ in order to reactivate the metabolic activity of the bacteria, then the ATP was measured using the BacTiter-Glo ${ }^{\mathrm{TM}}$ Microbial Cell Viability Assay kit (Promega, USA), which gives a luminescent signal in the presence of ATP. To that end, the bacterial solution was mixed with $100 \mu \mathrm{L}$ of the kit solution in a white 96-well plate and incubated for $5 \mathrm{~min}$ in the dark. Luminescence was subsequently measured in a micro-plate luminometer (BioTek, USA).

S. gordonii biofilms were evaluated by CFU counting. Thus, serial dilutions of the sonicated extract were performed in $0.9 \% \mathrm{NaCl}$ solution at $4{ }^{\circ} \mathrm{C}$, and $10 \mu \mathrm{L}$ of each dilution was plated on THB agar plates. These plates were incubated at $37^{\circ} \mathrm{C}$ for $16 \mathrm{~h}$, then the CFUs were counted. Oral microcosm biofilm model 769-NS contains at least 103 taxa and, in consequence, control of the incubation conditions in the agar plates for a thorough and reliable CFU count was not possible due to the different nutritional requirements and incubation time of the different strains. Alternatively, after microcosm biofilm sample collection, $500 \mu \mathrm{L}$ of bacterial extract was used for DNA extraction and purification using the MasterPure ${ }^{\mathrm{TM}}$ DNA Purification kit (Epicentre, Illumina, Inc., USA). The DNA concentration was then quantified using a Nanodrop 2000c spectrophotometer (Thermo Scientific, USA).

\subsection{Bacterial viability assay}

After incubation, the biofilms were stained with the FilmTracer ${ }^{\mathrm{TM}}$ LIVE/DEAD ${ }^{\mathrm{TM}}$ Biofilm Viability kit (Thermo Scientific, USA) and analyzed by confocal laser scanning 
microscopy (CLSM) with a multiphoton confocal microscope (A1R-HD, Nikon Instruments Inc., Japan). A 25X water immersion objective (Apo LWD, 1.1 NA) was used to directly visualize the biofilms in $0.9 \% \mathrm{NaCl}$ solution without a cover glass. Fluorescence emissions were collected at $488 \mathrm{~nm}$ for Syto9 (living cells) and at $561 \mathrm{~nm}$ for propidium iodide (dead cells). A $1024 \times 1024$ pixel $\left(0.12 \mu \mathrm{mpx}^{-1}\right)$ area was scanned, and the Z-stacks were recorded every $0.375 \mu \mathrm{m}$. Images were analyzed using the NIS-Elements Advanced Research analysis software (version 4.5, Nikon Corporation, Japan).

\subsection{Scanning electron microscopy}

After gentle removal of unattached bacteria, biofilms formed under static or dynamic conditions were fixed as described elsewhere. ${ }^{41}$ First, the biofilms on Ti discs were incubated in a primary fixation solution ( $2 \%$ glutaraldehyde and $0.15 \%$ Alcian blue in 0.1 M sodium cacodylate buffer, $\mathrm{pH} 7.4$ ), for $60 \mathrm{~min}$ at $\mathrm{RT}$. Then, after washing the discs in $0.1 \mathrm{M}$ sodium cacodylate buffer for $5 \mathrm{~min}$ at RT, the biofilms were treated with a secondary fixation solution $\left(1 \% \mathrm{OsO}_{4}\right.$ in $0.1 \mathrm{M}$ sodium cacodylate buffer) for $60 \mathrm{~min}$ at RT, washed and dehydrated using ethanol solutions with increasing concentration $(50 \%$, $70 \%, 80 \%, 95 \%$ and 100\%), critical point dried (Tousimis Samdi -780, USA) and coated

with a $50 \AA$ platinum layer. Finally, SEM images were recorded using a JEOL 6500 (JEOL USA, Inc., USA) field-emission gun scanning electron microscope (FEG-SEM).

\subsection{Cytocompatibility assay}

Human primary gingival fibroblasts (HGFs; PCS-201-018, ATCC, USA) were used to assess the cytocompatibility of the coated Ti discs. Cells were cultured for expansion in Fibroblast Basal Medium (PCS-201-030, ATCC, USA) supplemented accordingly (PCS201-041, ATCC, USA), with no phenol red or antibiotics, at $37{ }^{\circ} \mathrm{C}, 5 \% \mathrm{CO}_{2}$. Subsequently, cells were harvested and seeded on top of the Ti discs (6 mm diameter), 
previously placed in $24-w e l l$ plates, at 20,000 cells $\mathrm{cm}^{-2}$ (5655 cells per disc) in a $15-\mu \mathrm{L}$ drop. Samples were then incubated at $37^{\circ} \mathrm{C}$ for $3 \mathrm{~h}$ to allow cell attachment, and an excess of medium was added for further culture. After 1 and 3 days, samples were stained with the LIVE/DEAD ${ }^{\circledR}$ kit (Thermo Fisher Scientific, USA) following the manufacturer's instructions, and visualized upside-down with an inverted fluorescence microscope (Nikon Eclipse Ti-E coupled to a Nikon DS-2MBWc digital camera, Nikon Corporation, Japan) to confirm cell viability on the different coated Ti discs. Images were acquired and processed using the NIS-Elements Advanced Research software (version 4.5, Nikon Corporation, Japan).

\subsection{Statistical analysis}

A one-way analysis of variance (ANOVA) table with post-hoc Dunn's multiple comparison test was used to assess statistically significant differences between groups. A $p$-value of less than 0.05 was considered to be statistically significant $\left(* * p<0.001,{ }^{*} p\right.$ $<0.05)$. Results are shown as mean \pm standard deviation $(\mathrm{SD})(\mathrm{n} \geq 3)$.

\section{Results and discussion}

\subsection{Hybrid ELR design and synthesis}

Hybrid protein polymers (AMP-ELR) were based by a polycationic ELR [(VPGXG) 48 where $\mathrm{X}$ is $\mathrm{K}: \mathrm{V}$ in a 1:5 ratio] containing a $\mathrm{C}$-terminal Cys-Cys motif intended for sitespecific tethering to surfaces. L-GL13K was incorporated at the N-terminus of the ELR (named VC) using recombinant DNA techniques, thus resulting in the hybrid VCL. The orientation of the individual blocks of the hybrid VCL polymer was designed to optimize its recombinant expression without the addition of further amino acid residues to the AMP (Additional information can be found in the SI). This prevented the study of the effect of the orientation of the whole recombinant AMP-ELR molecules on the antimicrobial 
properties of the hybrid coatings, even though this has been proven to be a relevant feature of AMP coatings on different substrates recently. ${ }^{42-44}$ Both protein-engineered polymers (VC and VCL) were bioproduced in high yield as heterologous proteins in E. coli, followed by inverse transition cycling to obtain highly pure products with extreme control of their physicochemical properties. ${ }^{35}$ In contrast, chemical derivatization was needed to synthesize an ELR containing the D-enantiomer of the GL13K peptide (VCD) (Figure 1). Amino side groups of $\mathrm{VC}$ enabled covalent attachment of functional peptide motifs via a strain-promoted alkyne-azide cycloaddition (SPAAC) reaction. ${ }^{45}$ In this study, azidemodified D-GL13K peptides were tethered to cyclooctyne-modified amino groups of VC (VC-cyclo), which resulted in two to four D-GL13K peptides per molecule, as revealed by matrix-assisted laser desorption/ionization time-of-flight (MALDI-TOF) mass spectrometry (Figure S1, S2 and S3 and Table S1). The full amino acid sequences of the AMP, ELR and AMP-ELRs are listed in Table 1.

(a)

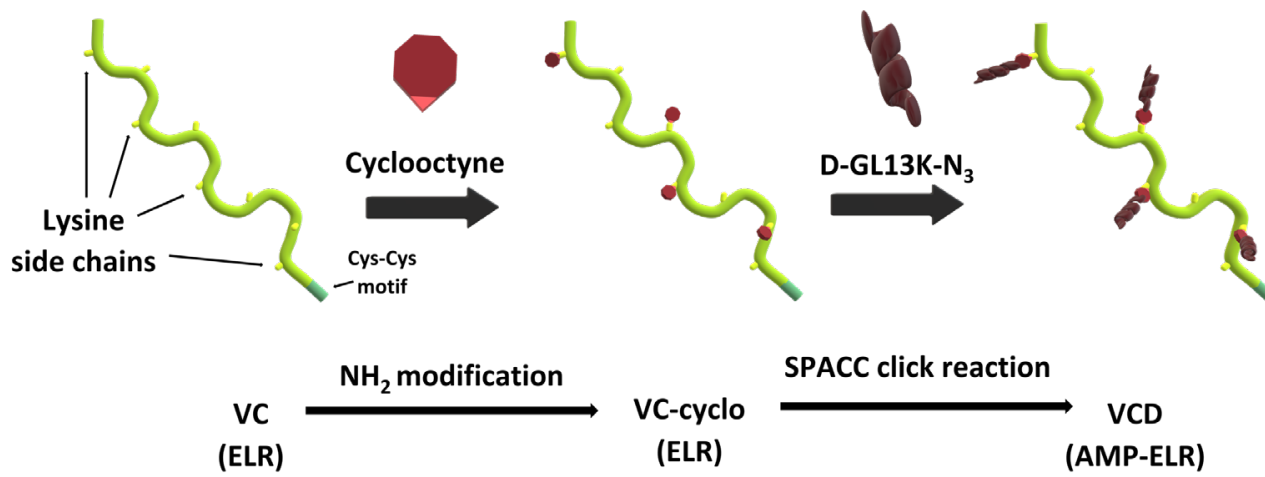

(b)<smiles>[R]CCNC(=O)OCC1[C@H]2CC=CC[C@H]12</smiles>

(c)

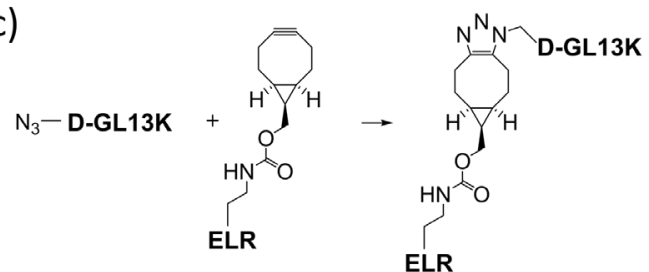

Figure 1. (a) Schematic representation of the covalent modification of the ELR (VC) with D-GL13K peptides to produce the hybrid AMP-ELR, VCD. Detailed schemes of (b) the cyclooctyne-modification of ELR and (c) the subsequent strain-promoted azide-alkyne cycloaddition (SPAAC). 
Table 1. Sequences and molecular weights (MW) of the AMP/ELR/AMP-ELRs used to manufacture covalent coatings on Ti substrates. The MW was calculated experimentally by MALDI-TOF.

\begin{tabular}{|c|c|c|c|}
\hline & Sequence & MW (Da) & $\begin{array}{c}\text { AMP } \\
\text { (no./molecule) }\end{array}$ \\
\hline D-GL13K & GkiiklkaslkIl-NH ${ }_{2}$ & 1424.0 & $\begin{array}{l}\text { D-GL13K } \\
\text { (1) }\end{array}$ \\
\hline VC & MESLLP VG (VPGVG VPGKG $\left.(\text { VPGVG })_{4}\right)_{8}$ VCC & 20819.8 & $\begin{array}{l}- \\
(0)\end{array}$ \\
\hline VCL & $\left.\mathrm{GKIIKLKASLKLLVLG}_{10} \mathrm{LVG}(\mathrm{VPGVGVPGKG(VPGVG})_{4}\right)_{8} \mathrm{VCC}$ & 22513.7 & $\begin{array}{l}\text { L-GL13K } \\
\text { (1) }\end{array}$ \\
\hline VCD & MESLLPVG(VPGVGVPGKG(VPGVG) $\left.)_{4}\right)_{8} V_{C C}$ & 26517.8 & $\begin{array}{c}\text { D-GL13K } \\
(2-4)\end{array}$ \\
\hline
\end{tabular}

\subsection{Coating fabrication and stability}

Covalent coatings of D-GL13K peptide or the ELR/AMP-ELRs were produced by immobilization with organosilanes on Ti surfaces, as shown schematically in Figure 2. Physicochemical (water contact angle (WCA), Figure 3 and S6) and chemical (X-ray photoelectron spectroscopy (XPS), Table 2 and Figure S7) characterization demonstrated the effectiveness of the surface functionalization.

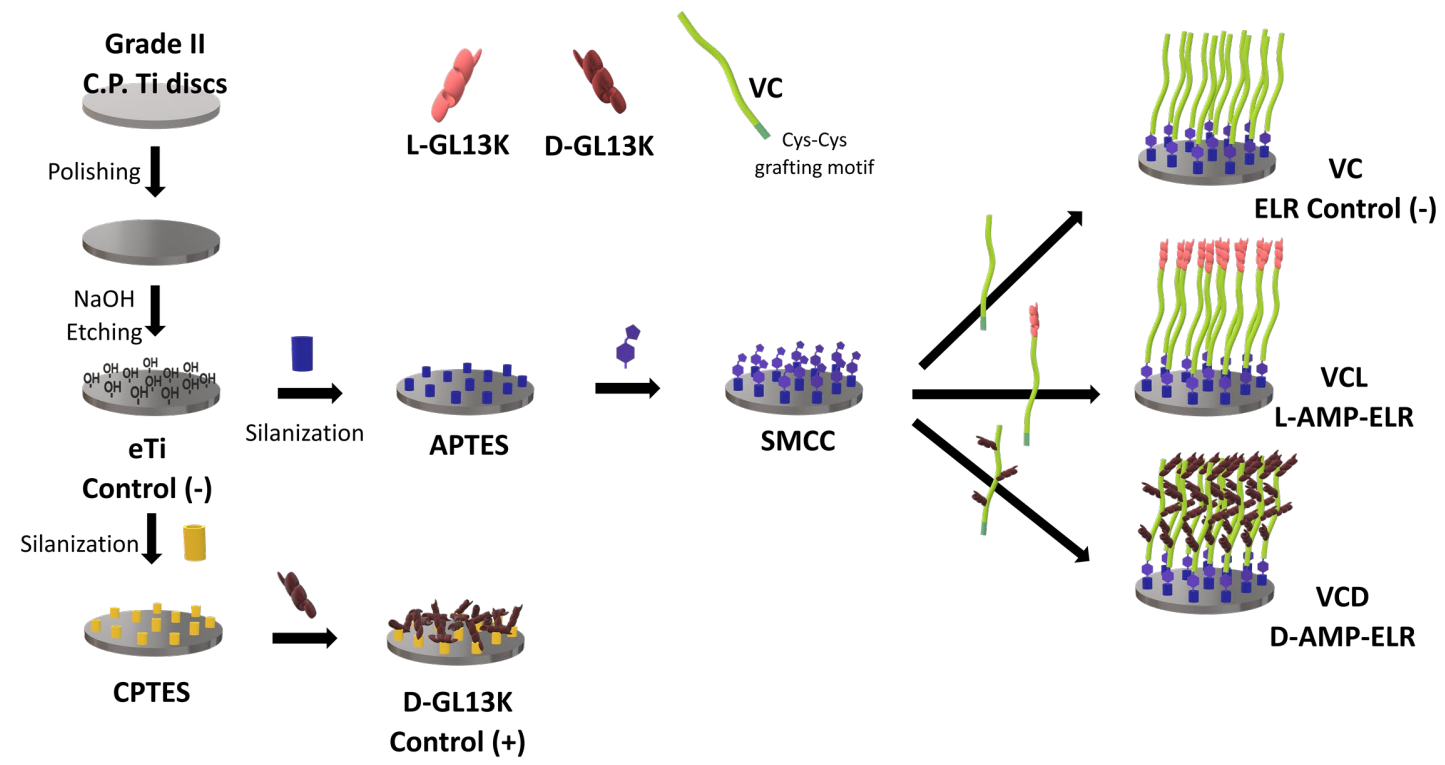

Figure 2. Schematic representation of the immobilization of D-GL13K peptides and the oriented tethering of proteinengineered polymers on titanium surfaces by titanium etching and silanization.

The $\mathrm{NaOH}$ etching of titanium (eTi) formed abundant polar hydroxyl groups on the metal surface, ${ }^{46}$ thus meaning that these surfaces were highly hydrophilic (WCA $=10.2^{\circ} \pm$ 
$4.2^{\circ}$ ). Silanization of the eTi surfaces with either CPTES or APTES significantly increased surface hydrophobicity $\left(\mathrm{WCA}=29^{\circ} \pm 0.7^{\circ}\right.$ and $\mathrm{WCA}=27.6^{\circ} \pm 3.6^{\circ}$ for $\mathrm{CPTES}$ and APTES, respectively). Immobilization of the D-GL13K peptides on CPTES-treated surfaces produced highly hydrophobic surfaces $\left(\mathrm{WCA}=123.4^{\circ} \pm 1.3^{\circ}\right)$. As mentioned previously, GL13K peptides are amphipathic molecules that re-structure and selfassemble under the solution conditions used herein. ${ }^{29}$ They then arrange at the surface of the metal so that the positively charged/hydrophilic groups of the free amines of the DGL13K molecule (4 lysines and the N-terminus) are attracted to, and react with, the negatively charged and hydrophilic surface of the CPTES-treated titanium. This molecular arrangement exposes the hydrophobic amino acids at the surface/air interface, which produces a highly hydrophobic surface. ${ }^{11,47,48}$

(a)

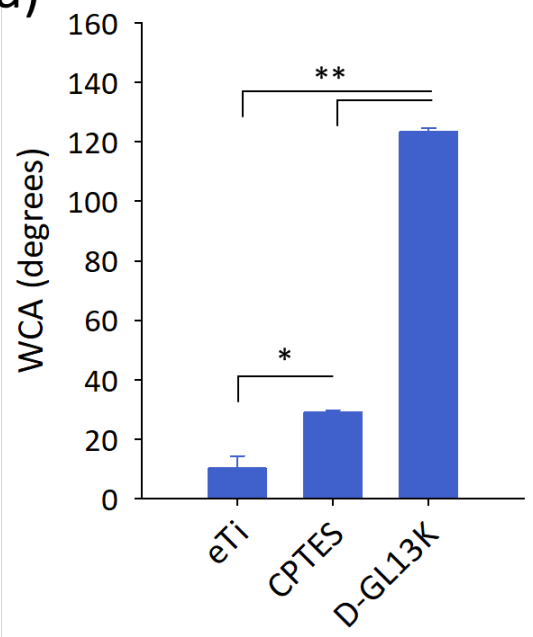

(c)

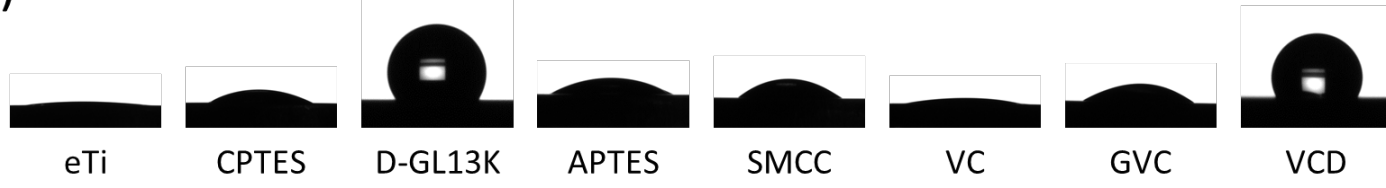

Figure 3. Water contact angle (WCA) of surfaces after different modification steps for covalent attachment of the AMP (a) or protein polymers (b). Bars represent the WCA after stabilization for $60 \mathrm{~s}$ and error bars represent standard deviations $(\mathrm{n}=6)$. (c) Representative images of the water drops on the different coatings $\left({ }^{*} p<0.05, * * p<0.001\right)$. 
In contrast, protein polymers were selectively immobilized on the surfaces via their Cterminal Cys-Cys motif. To that end bifunctional sulfo-SMCC was used as a linker between the APTES organosilane and the ELR/AMP-ELRs. Sulfo-SMCC deposition $\left(\mathrm{WCA}=39.9^{\circ} \pm 6.8^{\circ}\right)$ on APTES-silanized surfaces produced a moderate increase in surface hydrophobicity. Dynamic WCA analysis after immobilization of each of the three protein polymers (VC, VCL or VCD) on sulfo-SMCC treated surfaces revealed that the ELR-coated surfaces were hydrophobic $\left(\mathrm{WCA}>80^{\circ}\right)$ immediately after the water drop was deposited (Figure S6). VC- and VCL-coated surfaces exhibited drastic drops in WCA (increase in hydrophilicity) over time, whereas VCD-coated surfaces maintained their high hydrophobicity throughout the WCA analysis (100 s), with a final WCA of $104.5^{\circ}$ $\pm 9.7^{\circ}$, which is almost as high as that for D-GL13K coated surfaces. The amphipathic nature of the ELRs means that they are able to rearrange in response to contact with water molecules ${ }^{49}$ thus explaining the notable evolution of WCA for VC- and VCL-coated surfaces as they progressively expose their hydrophilic blocks at the solid/water interface. The difference in the final hydrophilicity between these two protein polymers reflects the effect of the presence and absence of L-GL13K in VCL $\left(\mathrm{WCA}=40.1^{\circ} \pm 5.7^{\circ}\right)$ and $\mathrm{VC}$ $\left(\mathrm{WCA}=11.2^{\circ} \pm 0.3^{\circ}\right)$, respectively. Chemical derivatization rendered a 'bottle brush' hybrid polymer (VCD) which contains between two and four D-GL13K peptides randomly located over the whole surface of the molecule, in comparison with the 'icing' architecture of the VCL (Figure 2). Thus, VCD-coated surfaces do not show major dynamic WCA changes. The interaction of the VCD-coating with water seems to be dominated by exposure of the ubiquitous peptide, as it is also the case for the D-GL13Kcoated surface. Importantly, divergences in the molecular architecture of the hybrid polymers prevent direct comparison of the coatings regarding their antimicrobial effects and mechanisms because AMP orientation and molecular architecture of the polymer 
seem to be relevant for coating properties. ${ }^{16}$ Therefore, future studies that address the direct comparison of hybrid AMP-ELR coatings with the same molecular architecture will be of value.

XPS analysis confirmed the successful modification of the Ti substrates (Table 2 and Figure S7). Thus, the Si $2 p$ peak increased after silanization of eTi with APTES or CPTES, which resulted in a marked increase in the $\mathrm{Si} / \mathrm{Ti}$ atomic ratio from 0.0003 (eTi surfaces) to 0.236 and 0.156 (for CPTES- and APTES-silanized surfaces, respectively), thus confirming the presence of these silanes on the Ti surface. In contrast, after peptide/protein modification, significant increases in $\mathrm{C} 1 \mathrm{~s}$ and $\mathrm{N} 1 \mathrm{~s}$ signals, and a substantial decrease in Ti $2 \mathrm{p}$ and Ti $2 \mathrm{~s}$, were observed. Quantitatively, the N/Ti atomic ratio increased from 0.012 (CPTES-silanized surfaces) to 1.972 after deposition of the DGL13K peptide and from 0.199 (SMCC-modified surfaces) to 5.416-5.948 for the ELR and AMP-ELRs (VC, VCL and VCD), thus demonstrating attachment of the peptide/protein coatings. Higher $\mathrm{N} / \mathrm{Ti}$ and $\mathrm{C} / \mathrm{Ti}$ atomic ratios were observed after deposition of the ELRs compared with the D-GL13K-modified surfaces. This was expected because of the larger molecular weight of the ELRs compared with the AMP.

Table 2. Quantitative XPS analysis of the titanium surfaces after different modification steps. Relative atomic ratios for the most representative elements $(\mathrm{Si}, \mathrm{N}$ and $\mathrm{C}$ ) in the coated molecules are shown with respect to the substrate element (Ti).

\begin{tabular}{llll}
\hline & $\mathbf{S i} / \mathrm{Ti}$ & $\mathbf{N} / \mathbf{T i}$ & $\mathbf{C} / \mathbf{T i}$ \\
\hline eTi & 0.0003 & 0.003 & 0.523 \\
CPTES & 0.236 & 0.012 & 1.456 \\
D-GL13K & 0.186 & 1.972 & 9.511 \\
APTES & 0.156 & 0.132 & 1.232 \\
SMCC & 0.145 & 0.199 & 3.327 \\
VC & 0.105 & 5.416 & 20.663 \\
VCL & 0.111 & 5.762 & 21.365 \\
VCD & 0.020 & 5.948 & 22.503 \\
\hline
\end{tabular}


We then tested the mechanical and thermal stability of the coatings. To evaluate the resistance to degradation, the functionalized $\mathrm{Ti}$ discs were ultrasonicated for $2 \mathrm{~h}$ in ultrapure water before being incubated in PBS $(1 \mathrm{X})$ at $37^{\circ} \mathrm{C}$ for 2 weeks. XPS and WCA analyses were performed after every challenge step. As shown in Figure 4a, the N/Ti atomic ratio decreased after ultrasonication in ultrapure water for all coatings. This was correlated with a slight increase in the Ti $2 p$ signal (Figure S8), indicative of the removal of small number of peptides/proteins from the functionalized surfaces. During incubation in $\mathrm{PBS}$, the $\mathrm{N} / \mathrm{Ti}$ and $\mathrm{C} / \mathrm{Ti}$ ratios remained stable in the D-GL13K coating, thus demonstrating its resistance to incubation under physiological conditions. In contrast, a progressive decrease in the $\mathrm{N} / \mathrm{Ti}$ and $\mathrm{C} / \mathrm{Ti}$ atomic ratios of the ELR/AMP-ELR coatings was observed during the first incubation week, with this stabilizing during the second incubation week (Figure 4a and S9).

(a)

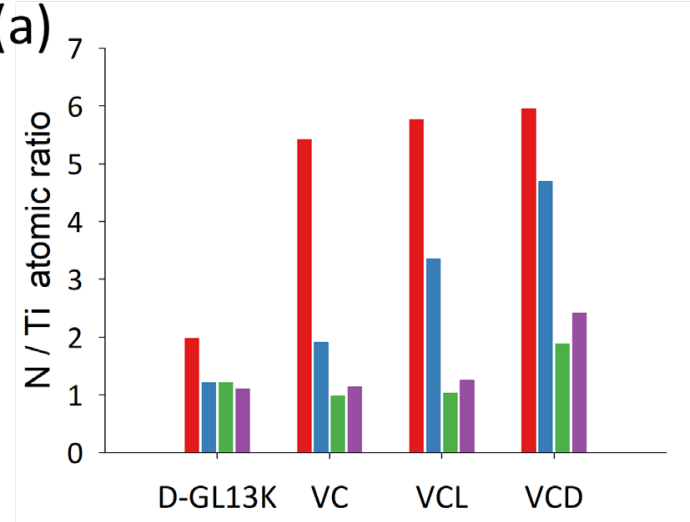

After immobilization + Ultrasonication (b)

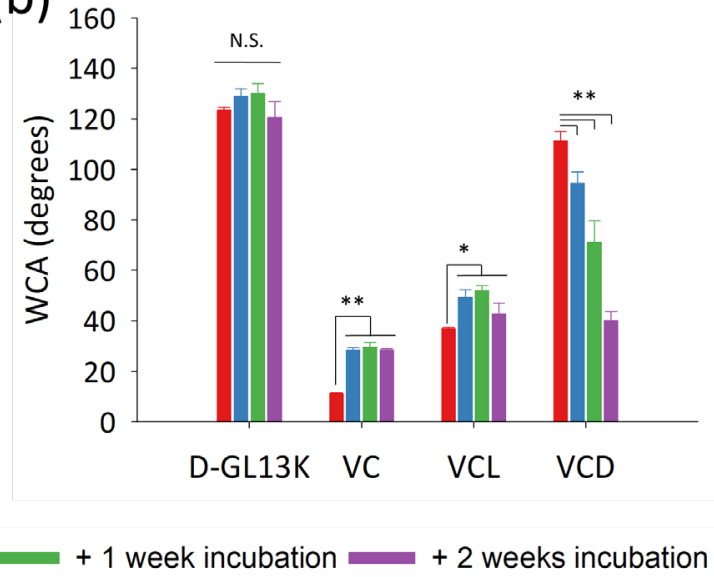

Figure 4. Physicochemical characterization of the coatings after the stability tests. Evolution of the N/Ti atomic ratio (a) and wettability (b) of the modified Ti surfaces after ultrasonication for 2 hours in aqueous solution, followed by incubation in PBS (1X) at $37^{\circ} \mathrm{C}$ for 1 and 2 weeks (N.S $=$ non-significant, ${ }^{*} p<0.05,{ }^{* *} p<0.001$ ).

Gradual removal of the protein polymers from the coatings may be due to their different degree of hydrophilicity and/or the chemical nature of the linker used for covalent immobilization. Thus, the D-GL13K peptide was nonspecifically attached to the CPTESsilanized surface via the free amines, thus exposing their apolar residues and producing 
highly hydrophobic coatings that provide impermeability to the surface. ${ }^{12}$ The high hydrophobicity of these coatings could protect the organosilanes from hydrolysis, thereby preventing peptide removal. All D-GL13K coatings remained highly hydrophobic after the challenges $\left(\mathrm{WCA}=120.6^{\circ} \pm 6.4^{\circ}\right)($ Figure $4 \mathrm{~b})$. Conversely, ELRs were tethered in an oriented way via their C-terminal Cys-Cys motif, thus enabling them to rearrange in solution and, consequently, resulting in a greater degree of interaction with water molecules, which may drive a greater degree of hydrolysis of the organosilanes and detachment of molecules from the coating comparing with the D-GL13K coating. In addition, as can be seen from Figure $3 b, \mathrm{VC}$ and VCL coatings were more hydrophilic than the D-GL13K coating. Finally, although VCD immobilization produced hydrophobic surfaces, a similar behavior to VC and VCL was observed. However, it is worth noting that the WCA for the VCD coating remained significantly higher than for the VC coating (ELR control) after the challenges $(p<0.001)$, thus suggesting that DGL13K peptides were still present in the coating.

\subsection{Antibiofilm activity against monospecies biofilm}

Functionalized Ti surfaces were tested against $S$. gordonii biofilms under dynamic conditions in a DFBR to assess the antibiofilm capacity of the coatings. S. gordonii bacteria are relevant early colonizers in dental plaque that enable the attachment of other pathogenic bacteria in the biofilm, such as $P$. gingivalis ${ }^{33}$ After incubation, LIVE/DEAD images of the biofilms (Figure 5a) revealed that eTi discs were completely covered by $S$. gordonii biofilm, while D-GL13K coatings provided antibiofilm and bactericidal activity to the surface, as reported previously. ${ }^{11}$ ELR coatings also hindered biofilm maturation. The VC coating (ELR control) prevented the formation of a mature biofilm but with no bactericidal effect due to a low-fouling effect. ${ }^{35}$ However, the combination of GL13K peptides (L or D enantiomers) with the VC polymer in the hybrid coatings (VCL and 
VCD) provided additional bactericidal activity against the bacteria remaining on the surface.

SEM evaluation (Figure 5b, 5c and S10) confirmed the antibiofilm activity of the coatings against $S$. gordonii. Thus, eTi discs were covered by a thick biofilm, which fully prevented visualization of the Ti substrate underneath the bacteria, whereas all coatings hindered biofilm growth, as shown in the SEM images, by decreasing bacterial colonization of the surfaces in comparison to the eTi control substrate. Consistent with previous findings, ${ }^{11}$ bacteria with morphological changes were found on the coatings containing AMPs (D-GL13K, VCL and VCD). This may be indicative of the effects of the AMP on $S$. gordonii cell walls and/or membranes. Thus, ruptured bacteria and/or elongated bacteria were found on the positive control (D-GL13K) and on the hybrid AMP-ELR (VCL and VCD) coated surfaces. 


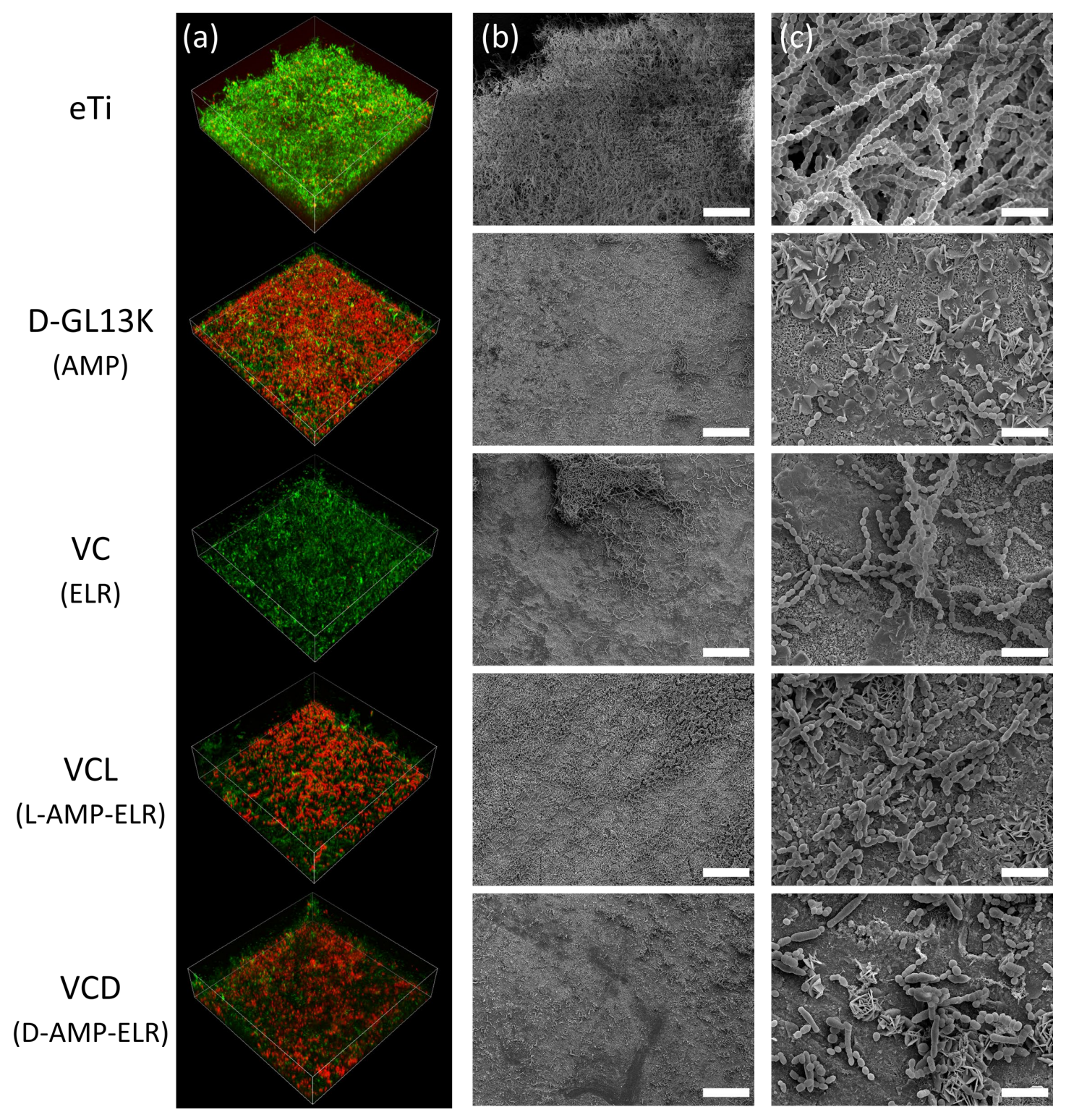

Figure 5. S. gordonii biofilms formed on the surfaces after incubation under dynamic conditions (DFBR): (a) LIVE/DEAD staining images of the streptococcal biofilms obtained by confocal laser scanning microscopy (CLSM). Green cells correspond to bacteria with intact membranes, whereas the bacterial membranes are compromised or damaged in red bacteria (area xy $=126 \times 126 \mu \mathrm{m}$ ). (b) and (c) SEM micrographs of the immobilized biofilms on the surfaces. After $48 \mathrm{~h}$ incubations, coatings prevented the grow of a mature biofilm on the surfaces, whereas eTi surfaces (control) were covered by multiple layers of bacteria and extracellular matrix that rendered an irregular S. gordonii biofilm. General view of the biofilms (b) shows the irregularities and multiple heights of the biofilms formed on control eTi surfaces (blank zone in the upper-right corner corresponds with low thickness region). Scale bars $=40 \mu \mathrm{m}(\mathrm{b})$ and $4 \mu \mathrm{m}(\mathrm{c})$.

These results, together with the physical characterization, indicate that ELRs serve as a functional platform for AMP-tethering. ELR-coatings showed dynamic response and they allowed proper folding of the AMPs; hence, the peptides were active within the recombinant polymer (Figure 5). Recent studies evidenced that the antimicrobial potential of amphiphilic AMPs is related to their ability to adopt secondary conformations with 
high content in $\beta$-sheets. ${ }^{29,48,50}$ Thus, polymeric platforms should enable the functional folding of the AMPs. The tendency to fold into $\beta$-sheet structures is associated with an overexposure of the hydrophobic residues and enhanced interaction with bacterial walls and membranes. ${ }^{32,48,51}$ In this regard, hybrid coatings showed an increased hydrophobicity comparing with the control VC-coatings in addition to bactericidal activity, demonstrating in this way the functional integration of the AMPs within the proteinengineered polymers.

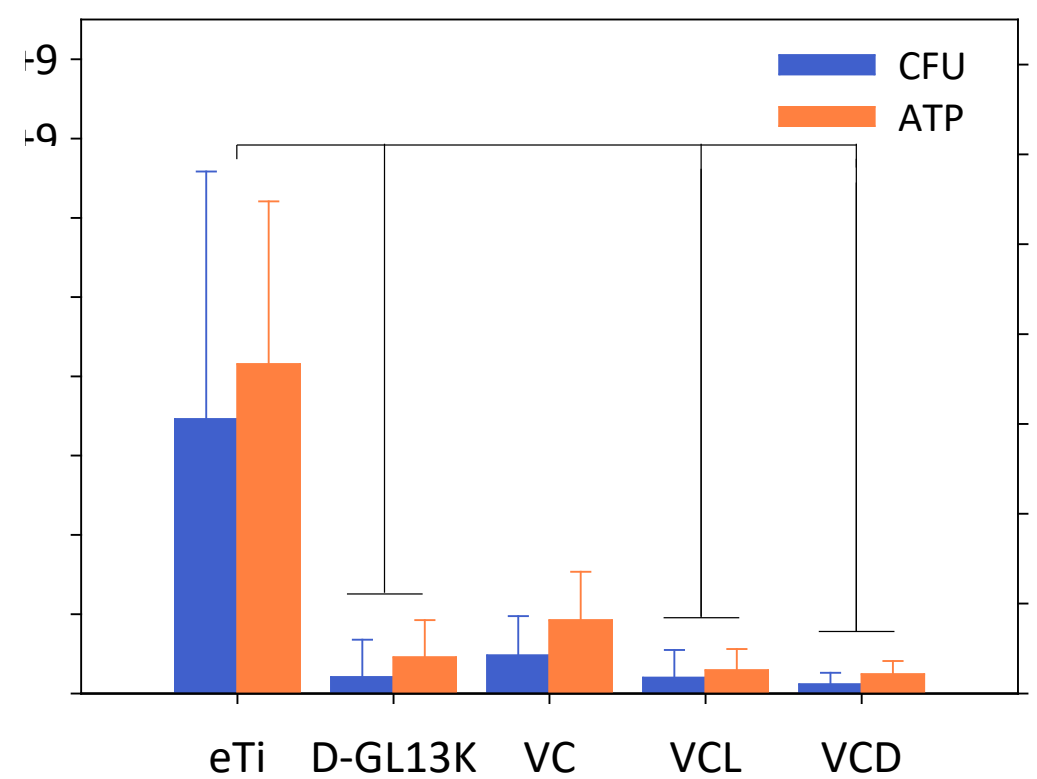

Figure 6. Quantification of antibiofilm activity by CFU counting and metabolic activity assay, as measured after incubation for $48 \mathrm{~h}$ under dynamic conditions. The presence of the GL13K (L or D enantiomer) in the hybrid ELR coatings significantly decreased biofilm formation with respect to uncoated eTi $\left({ }^{*} p<0.05\right)$.

Furthermore, quantification of the antibiofilm activity of the coatings was successfully assessed by CFU counting and evaluation of the metabolic activity of the remaining biofilms after the incubation period (Figure 6). In comparison to eTi discs, all coatings reduced biofilm formation, with significantly lower CFU and ATP values. VC coatings showed a notable reduction in bacteria, as shown in Figure 5, but the CFU and ATP values revealed that the antimicrobial effect of these coatings was moderate and lower than for 
all other coatings. ELR-based coatings prevented nonspecific protein adsorption, ${ }^{26}$ thus conferring antifouling properties on the surfaces, ${ }^{49}$ that may hamper bacterial attachment and biofilm formation under static conditions, as described previously for other Gram positive bacteria. ${ }^{35}$ Nevertheless, this low-fouling activity of VC coatings was unable to significantly reduce $S$. gordonii biofilm development under dynamic conditions compared with eTi substrates. In contrast, hybrid AMP-ELR coatings (VCL and VCD), with additional bactericidal activity conferred by the AMPs, significantly decreased biofilm formation on the surfaces compared to eTi, which is similar to the result obtained with D-GL13K coatings.

\subsection{Antibiofilm activity against oral microcosm biofilms}

Following successful assessment of the antimicrobial effects of the hybrid AMP-ELR coatings against $S$. gordonii biofilms, we tested their antibiofilm potential by applying oral-simulating and more challenging in vitro conditions. Thus, a well characterized oral microcosm biofilm model collected from cariogenic patients ${ }^{34}$ was grown onto the hybrid coatings (VCL and VCD) using a DFBR. eTi and D-GL13K-coated surfaces were also tested in order to compare the efficacy of the hybrid coatings.

After incubation of the surfaces with the oral microcosm biofilm model in the DFBR, under dynamic conditions, for 6 days, evaluation of the biofilms by confocal laser scanning microscopy (CLSM) showed that a thick complex biofilm covered the whole surface of the eTi discs (Figure 7a). In contrast, all coatings tested hampered biofilm formation and/or growth, but with substantial differences in the way they did so. Thus, biofilm thickness was decreased on VCL coatings compared to eTi surfaces, but the coatings with this hybrid ELR exhibited no discernable bactericidal activity. On the other hand, coatings that included immobilized molecules containing D-GL13K peptides, that is D-GL13K and VCD coatings, exhibited both a decrease in the thickness of the biofilm 
in comparison to eTi surfaces and bactericidal activity against the bacteria that remained on the surface, as shown in the CLSM images. Additionally, these results indirectly evidenced the enzymatic resistance of the coatings. After 6 days of incubation, the coatings maintained their antimicrobial potential, which demonstrated the resistance of the coatings to be degraded by oral microbial communities. Further studies with longer challenge periods and more controlled conditions in different environmental and biological conditions will be relevant in the future to assess the full translational potential of the coatings.
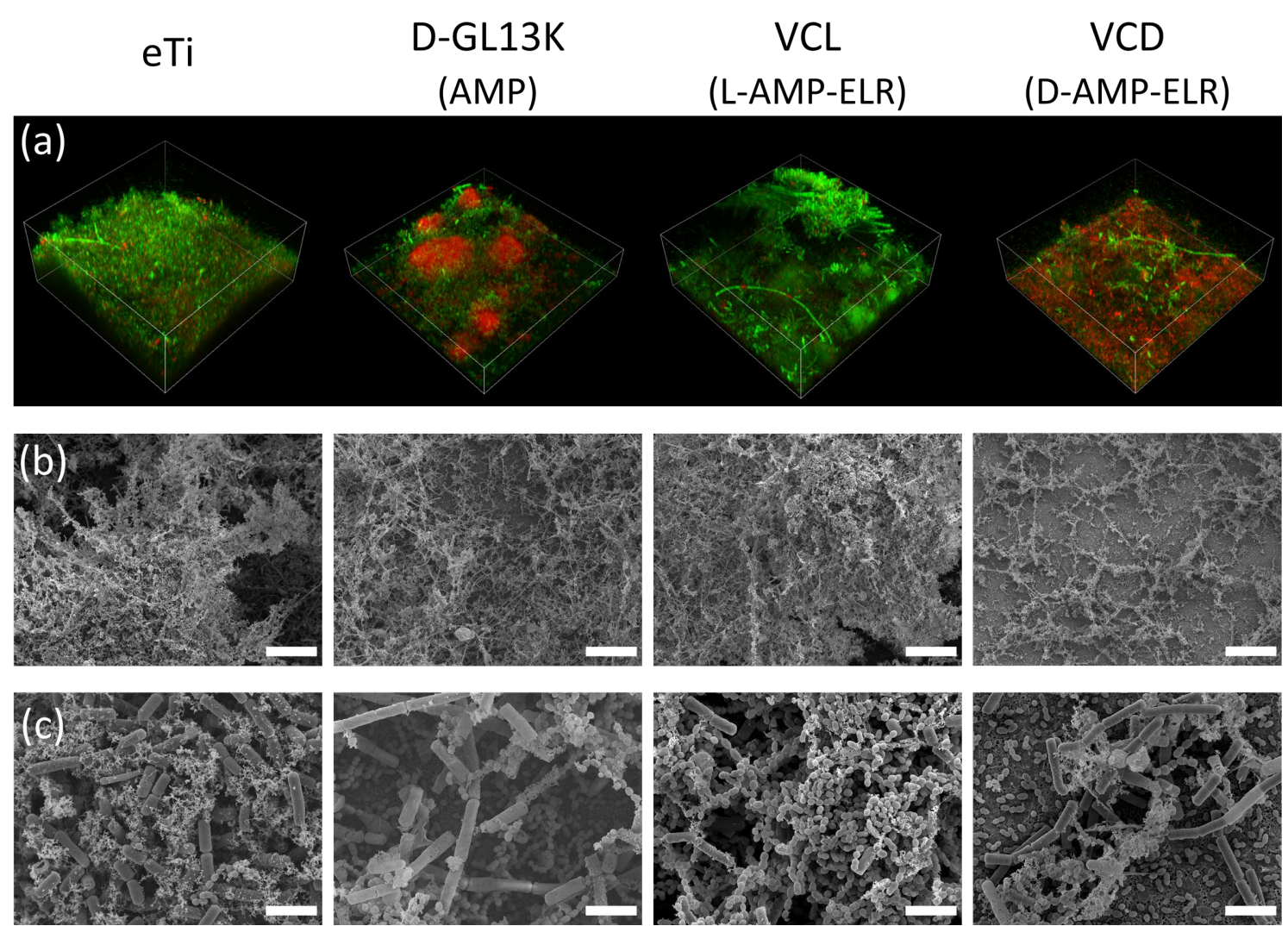

Figure 7. Representative CLSM and SEM images of oral microcosm biofilms after incubation in the DFBR for 6 days. (a) CLSM images of the biofilm with LIVE/DEAD staining (xy section $=126 \times 126 \mu \mathrm{m}$ ). SEM micrographs: (b) general view (scale bar $=40 \mu \mathrm{m}$ ) and (c) magnified images of the biofilms (scale bar $=4 \mu \mathrm{m}$ ). eTi discs were found to be completely covered by a thick biofilm. Although all the coatings prevented the development of a mature biofilm, bactericidal activity was only found for those coatings containing D-GL13K peptides (D-GL13K and VCD).

SEM micrographs (Figure $7 b, c)$ confirmed the strong antibiofilm activity of the immobilized D-GL13K peptide coatings against oral microcosm biofilms, either alone (D-GL13K coating) or via the ELR (VCD coating). The presence of the D-enantiomeric 
form of the GL13K peptide in the coatings prevented the formation of a mature biofilm and provoked cell wall damage in bacteria that remained on the surfaces (Figure 8). A continuous microcosm biofilm was formed on both eTi and VCL surfaces, but the biofilm was thinner on VCL coatings than on eTi surfaces (Figure 7). However, there were no significant differences in either metabolic activity (luminescence intensity) or DNA content between the bacteria remaining on the eTi surfaces and the VCL coatings (Figure 9).

Recently, it has been demonstrated that the L-enantiomer of the GL13K peptide is less potent against Gram (+), Gram (-) bacteria and bacterial microcosms, ${ }^{29,48}$ and more sensitive to bacterial proteases, than the D-enantiomer. ${ }^{32}$ Although studies suggested that AMP-mimicking $\beta$-peptide polymer-coatings are likely to induce bacteria death via the depletion of divalent cations $\left(\mathrm{Mg}^{2+}\right.$ and $\left.\mathrm{Ca}^{2+}\right),{ }^{52}$ the molecular mechanism of killing with GL13K peptides has been related to the direct interaction between the peptides and the bacterial surface components. Thus, the greater tendency of the D-enantiomer to fold into secondary $\beta$ structures ${ }^{29,48}$ seems to enhance the affinity to these components, including teichoic acids and lipopolysaccharides (LPS), ${ }^{32,53}$ that may result in the disruption of the bacterial wall or LPS outer membrane. In this sense, VCL is a full L-amino acid polypeptide due to its recombinant synthesis. Despite the antibiofilm potency of VCL against $S$. gordonii biofilms, its antimicrobial activity was limited to impeding surface colonization and formation of a biofilm with a more complex microbiota. In contrast, the presence of the D-enantiomer of the GL13K peptide in the coatings inhibited biofilm formation by more than $96 \%$ in terms of metabolic activity compared to eTi surfaces, irrespective of the manner of presentation of these D-GL13K peptides. Total DNA quantification provided an estimation of the total number of bacteria remaining on the surface. In comparison with the eTi control, D-GL13K and VCD coatings caused a 
significant reduction in the total remaining bacteria (a decrease of $65 \%$ and $70 \%$, respectively). This demonstrates the efficacy of VCD coatings in the prevention of complex oral biofilm growth under dynamic conditions. Consequently, these results suggest the potential application of ELRs as multivalent platforms for AMPs in the development of ECM-mimicking coatings for indwelling devices.
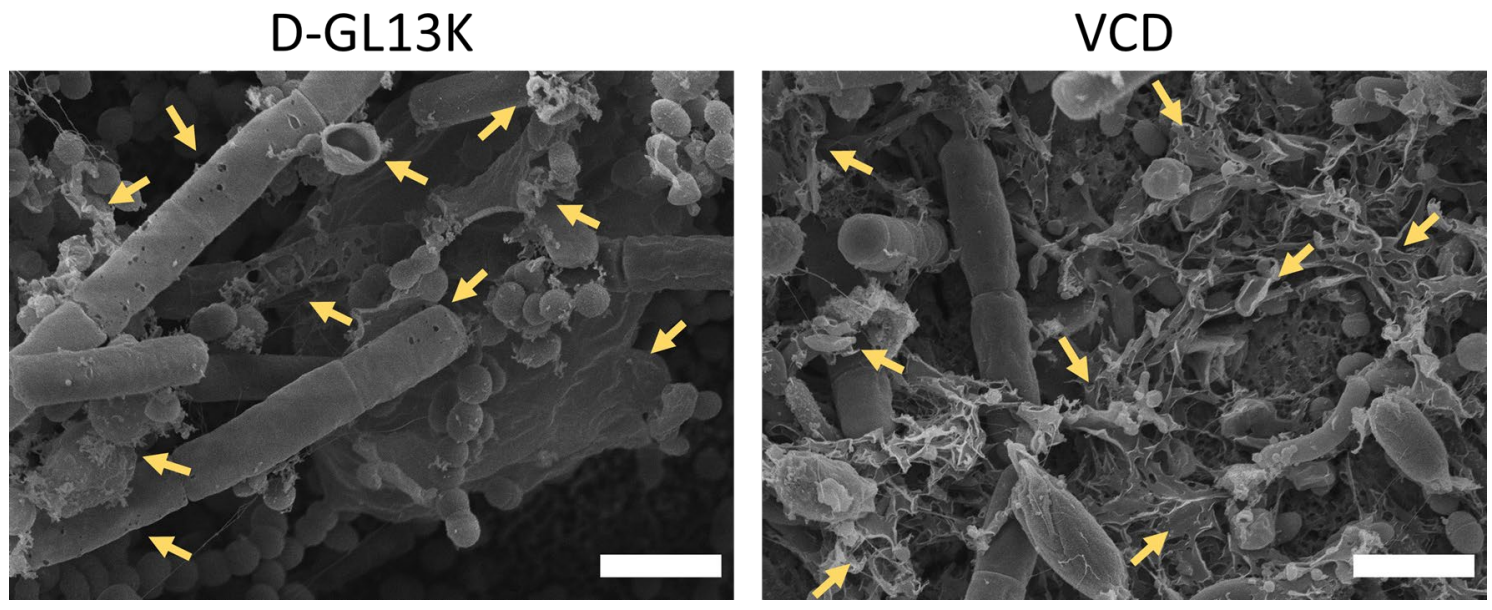

Figure 8. SEM micrographs of the disrupted bacteria (yellow arrows) found in the surfaces containing D-GL13K peptides. Scale bar $=2 \mu \mathrm{m}$.

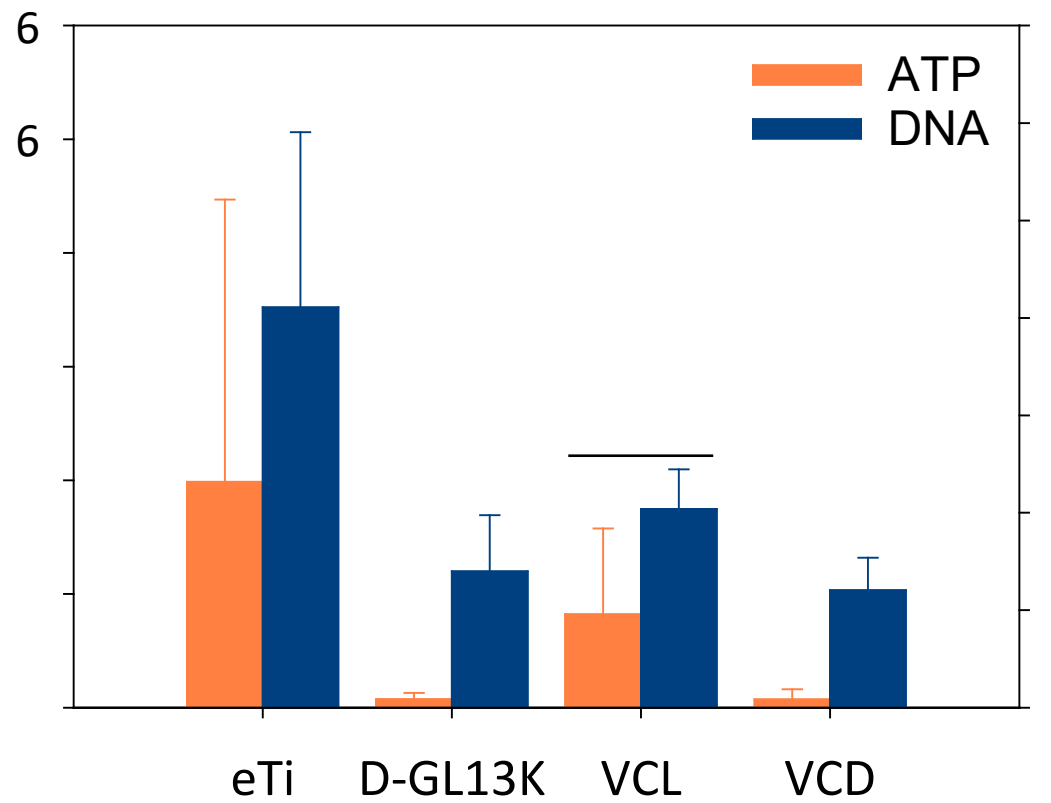

Figure 9. Antibiofilm activity of the coatings against oral microcosm biofilms after incubation in the DFBR for 6 days. Metabolic activity and DNA quantification demonstrated the strong and significant antibiofilm activity of the coatings (N.S. $=$ non-significant, ${ }^{*} p<0.05, * * p<0.001$ compared with eTi control). 


\subsection{Cytocompatibility of the antimicrobial coatings}

The antibiofilm coatings described here will potentially be used in dental applications. As such, it was of interest to assess their cytocompatibility with mammalian cells, more specifically with those present in the oral cavity. We therefore evaluated the viability of primary human gingival fibroblasts (HGFs) cultured on coated Ti discs, in comparison with the uncoated eTi. LIVE/DEAD results showed that the HGFs cultured on Ti discs covered with the different peptides and ELRs were nearly $100 \%$ viable and proliferated appropriately during culture for up to 3 days (Figure 10). This is in agreement with previous findings regarding the cyto- and biocompatibility of ELRs and the low toxicity of the GL13K peptide. ${ }^{48,54,55}$ Overall, these results indicate that the antimicrobial coatings are likely to be safe for dental applications.
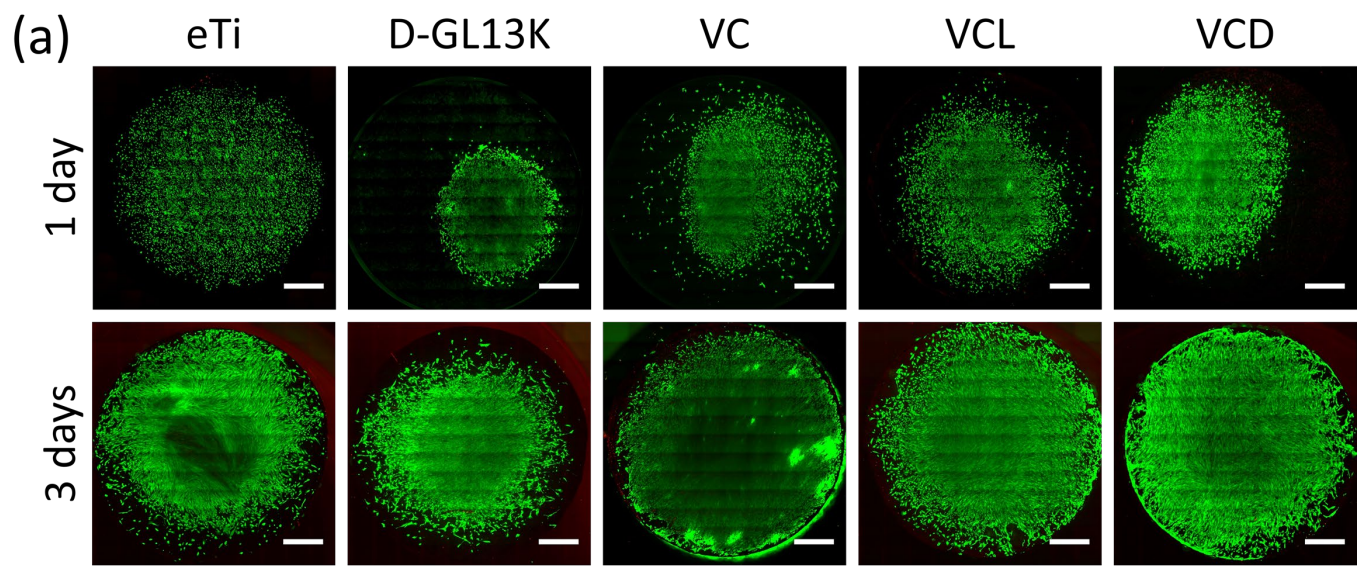

(b)

eTi
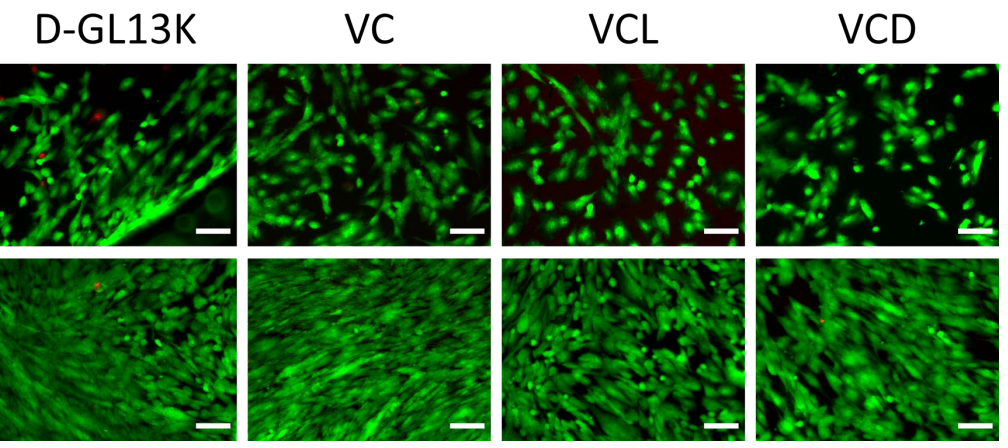

Figure 10. HGFs on the Ti surfaces with LIVE/DEAD staining after incubation for 1 and 3 days. (a) Composite images of whole Ti discs (scale bar $=1 \mathrm{~mm}$ ) and (b) magnified pictures of specific fields (scale bar $=100 \mu \mathrm{m}$ ), showing living (green) and dead (red) cells. 


\section{Conclusions}

We have designed a protein-engineered molecular platform for AMPs based on a ECMmimicking polymer for the development of antibiofilm and cytocompatible coatings on titanium that can prevent IAIs. We have also demonstrated that hybrid coatings based on VCD paralleled the strong antimicrobial activity of D-GL13K coatings against both $S$. gordonii and oral microcosm biofilms when tested using challenging dynamic biofilm culturing conditions and, hence, their potential use as multivalent coatings. Proteinengineered polymers enable the integration of multiple bioactivities with exquisite control and, hence, the achievement of coatings with increased biofunctionalities. Molecular systems that combine AMPs with protein-engineered polymers may enable the development of safe multifunctional coatings for implants and other materials used in regenerative medical applications.

\section{Acknowledgements}

The authors would like to thank Professor Joel Rudney (University of Minnesota) for facilitating the use of microbiology facilities and the S. gordonii bacteria and oral bacterial microcosms used here and Professor Sven Gorr (University of Minnesota) for facilitating the use of his lab facilities. The authors also acknowledge Ms. Ruoqiong Chen for technical assistance with the drip-flow biofilm bioreactor and antimicrobial tests. Parts of this work were carried out in the Characterization Facility, University of Minnesota, which receives partial support from NSF through the MRSEC program. CLSM was performed at the University of Minnesota Imaging Centers (http://uic.umn.edu) with the assistance of Dr. Guillermo Marques. The authors are grateful for funding from the European Commission (NMP-2014-646075), the Spanish Government (MAT201678903-R and PCIN-2015-010 (FunBioPlas)), the Junta de Castilla y León (VA317P18) and the Centro en Red de Medicina Regenerativa y Terapia Celular de Castilla y León. 


\section{References}

1 H. J. Busscher, H. C. Van Der Mei, G. Subbiahdoss, P. C. Jutte, J. J. A. M. Van Den Dungen, S. A. J. Zaat, M. J. Schultz and D. W. Grainger, Sci. Transl. Med., 2012, 4, 153rv10-153rv10.

2 S. S. Magill, J. R. Edwards, W. Bamberg, Z. G. Beldavs, G. Dumyati, M. A. Kainer, R. Lynfield, M. Maloney, L. McAllister-Hollod, J. Nadle, S. M. Ray, D. L. Thompson, L. E. Wilson, S. K. Fridkin and Emerging Infections Program Healthcare-Associated Infections and Antimicrobial Use Prevalence Survey Team, N. Engl. J. Med., 2014, 370, 1198-1208.

3 R. O. Darouiche, N. Engl. J. Med., 2004, 350, 1422-1429.

4 R. O. Darouiche and R. O. Darouiche, Clin. Infect. Dis., 2001, 33, 1567-1572.

$5 \quad$ World Health Organization, Antimicrobial resistance: global report on surveillance, 2014.

6 R. E. W. Hancock and H. G. Sahl, Nat. Biotechnol., 2006, 24, 1551-1557.

$7 \quad$ K. A. Brogden, Nat. Rev. Microbiol., 2005, 3, 238-250.

8 J. D. Steckbeck, B. Deslouches and R. C. Montelaro, Expert Opin. Biol. Ther., 2014, 14, 11-4.

9 F. Costa, P. Gomes and M. C. L. Martins, in Peptides and Proteins as Biomaterials for Tissue Regeneration and Repair, Woodhead Publishing, 2018, pp. 329-345.

10 K. V. Holmberg, M. Abdolhosseini, Y. Li, X. Chen, S.-U. Gorr and C. Aparicio, Acta Biomater., 2013, 9, 8224-8231.

11 X. Chen, H. Hirt, Y. Li, S. U. Gorr and C. Aparicio, PLoS One, 2014, 9, e111579.

12 D. G. Moussa, A. Fok and C. Aparicio, Acta Biomater., 2019, 88, 251-265.

13 H. Yazici, M. B. O’Neill, T. Kacar, B. R. Wilson, E. E. Oren, M. Sarikaya and C. Tamerler, ACS Appl. Mater. Interfaces, 2016, 8, 5070-5081.

14 D. T. Yucesoy, M. Hnilova, K. Boone, P. M. Arnold, M. L. Snead and C. Tamerler, JOM, 2015, 67, 754-766.

15 M. Bagheri, M. Beyermann and M. Dathe, Antimicrob. Agents Chemother., 2009, 53, 1132-1141.

16 M. Salwiczek, Y. Qu, J. Gardiner, R. A. Strugnell, T. Lithgow, K. M. McLean and H. Thissen, Trends Biotechnol., 2014, 32, 82-90.

17 C. Wisdom, S. K. VanOosten, K. W. Boone, D. Khvostenko, P. M. Arnold, M. L. Snead and C. Tamerler, J. Mol. Eng. Mater., 2016, 04, 1640005.

18 E. C. Wisdom, Y. Zhou, C. Chen, C. Tamerler and M. L. Snead, ACS Biomater. Sci. Eng., 2019, acsbiomaterials.9b01213.

19 S.-X. Xie, L. Song, E. Yuca, K. Boone, R. Sarikaya, S. K. VanOosten, A. Misra, Q. Ye, P. Spencer and C. Tamerler, ACS Appl. Polym. Mater., 2020, 
acsapm.9b00921.

20 K. Yu, J. C. Y. Lo, M. Yan, X. Yang, D. E. Brooks, R. E. W. Hancock, D. Lange and J. N. Kizhakkedathu, Biomaterials, 2017, 116, 69-81.

21 S. Atefyekta, M. Pihl, C. Lindsay, S. C. Heilshorn and M. Andersson, Acta Biomater., 2019, 83, 245-256.

22 S. Roberts, M. Dzuricky and A. Chilkoti, FEBS Lett., 2015, 589, 2477-2486.

23 D. W. Urry, T. M. Parker, M. C. Reid and D. C. Gowda, J. Bioact. Compat. Polym., 1991, 6, 263-282.

24 R. L. DiMarco and S. C. Heilshorn, Adv. Mater., 2012, 24, 3923-3940.

25 G. Ciofani, G. G. Genchi, I. Liakos, A. Athanassiou, V. Mattoli and A. Bandiera, Acta Biomater., 2013, 9, 5111-5121.

26 E. Salvagni, G. Berguig, E. Engel, J. C. Rodriguez-Cabello, G. Coullerez, M. Textor, J. A. Planell, F. J. Gil and C. Aparicio, Colloids Surfaces B Biointerfaces, 2014, 114, 225-233.

27 Y. Li, X. Chen, A. J. Ribeiro, E. D. Jensen, K. V. Holmberg, J. C. RodriguezCabello and C. Aparicio, Adv. Healthc. Mater., 2014, 3, 1638-1647.

28 L. Li, C.-K. Mo, A. Chilkoti, G. P. Lopez and N. J. Carroll, Biointerphases, 2016, 11, 021009.

29 Z. Ye, X. Zhu, S. Acosta, D. Kumar, T. Sang and C. Aparicio, Nanoscale, 2019, 11, 266-275.

30 N. Harmouche, C. Aisenbrey, F. Porcelli, Y. Xia, S. E. D. Nelson, X. Chen, J. Raya, L. Vermeer, C. Aparicio, G. Veglia, S.-U. Gorr and B. Bechinger, Biochemistry, 2017, 56, 4269-4278.

31 M. Abdolhosseini, S. R. Nandula, J. Song, H. Hirt and S.-U. Gorr, Peptides, 2012, 35, 231-238.

32 H. Hirt, J. W. Hall, E. Larson and S.-U. Gorr, PLoS One, 2018, 13, e0194900.

33 B. Rosan and R. J. Lamont, Microbes Infect., 2000, 2, 1599-1607.

34 J. D. Rudney, R. Chen, P. Lenton, J. Li, Y. Li, R. S. Jones, C. Reilly, A. S. Fok and C. Aparicio, J. Appl. Microbiol., 2012, 113, 1540-53.

35 S. Acosta, L. Quintanilla, M. Alonso, C. Aparicio and J. C. Rodríguez-Cabello, ACS Biomater. Sci. Eng., 2019, 5, 4708-4716.

36 I. González De Torre, M. Santos, L. Quintanilla, A. Testera, M. Alonso and J. C. Rodríguez Cabello, Acta Biomater., 2014, 10, 2495-2505.

37 C. Fenoll-Palomares, J. V Muñoz Montagud, V. Sanchiz, B. Herreros, V. Hernández, M. Mínguez and A. Benages, Rev. Esp. Enferm. Dig., 2004, 96, $773-$ 83.

38 J. D. Rudney, P. D. Jagtap, C. S. Reilly, R. Chen, T. W. Markowski, L. Higgins, J. E. Johnson and T. J. Griffin, Microbiome, 2015, 3, 69.

39 C. Dawes, J. Physiol., 1972, 220, 529-45. 
40 A. J. McBain, C. Sissons, R. G. Ledder, P. K. Sreenivasan, W. De Vizio and P. Gilbert, J. Appl. Microbiol., 2005, 98, 624-634.

41 R. Alink and D. Gerteisen, in PEM Fuel Cell Diagnostic Tools, John Wiley \& Sons, Inc., Hoboken, NJ, USA, 2011, vol. 25, pp. 315-332.

42 K. Rapsch, F. F. Bier, M. Tadros and M. Von Nickisch-Rosenegk, Bioconjug. Chem., 2014, 25, 308-319.

43 F. M. T. A. Costa, S. R. Maia, P. A. C. Gomes and M. C. L. Martins, Biomaterials, 2015, 52, 531-538.

44 M. Barbosa, F. Costa, C. Monteiro, F. Duarte, M. C. L. Martins and P. Gomes, Acta Biomater., 2019, 84, 242-256.

45 T. Flora, I. G. de Torre, M. Alonso and J. C. Rodríguez-Cabello, J. Mater. Sci. Mater. Med., 2019, 30, 30.

46 H.-M. Kim, F. Miyaji, T. Kokubo and T. Nakamura, J. Biomed. Mater. Res., 1996, 32, 409-417.

47 P. Sevilla, J. Gil and C. Aparicio, IRBM, 2017, 38, 256-265.

48 D. G. Moussa, J. A. Kirihara, Z. Ye, N. G. Fischer, J. Khot, B. A. Witthuhn and C. Aparicio, J. Dent. Res., 2019, 98, 1112-1121.

49 M. Pierna, M. Santos, F. J. Arias, M. Alonso and J. C. Rodríguez-Cabello, Biomacromolecules, 2013, 14, 1893-1903.

50 G. Manzo, M. A. Scorciapino, P. Wadhwani, J. Bürck, N. Pietro Montaldo, M. Pintus, R. Sanna, M. Casu, A. Giuliani, G. Pirri, V. Luca, A. S. Ulrich and A. C. Rinaldi, PLoS One, 2015, 10, e0116379.

51 V. Balhara, R. Schmidt, S.-U. Gorr and C. DeWolf, Biochim. Biophys. Acta Biomembr., 2013, 1828, 2193-2203.

52 Y. Qian, F. Qi, Q. Chen, Q. Zhang, Z. Qiao, S. Zhang, T. Wei, Q. Yu, S. Yu, Z. Mao, C. Gao, Y. Ding, Y. Cheng, C. Jin, H. Xie and R. Liu, ACS Appl. Mater. Interfaces, 2018, 10, 15395-15400.

53 H. Hirt and S.-U. Gorr, Antimicrob. Agents Chemother., 2013, 57, 4903-4910.

54 A. Ibáñez-Fonseca, T. L. Ramos, I. González de Torre, L. I. Sánchez-Abarca, S. Muntión, F. J. Arias, M. C. del Cañizo, M. Alonso, F. Sánchez-Guijo and J. C. Rodríguez-Cabello, J. Tissue Eng. Regen. Med., 2018, 12, e1450-e1460.

55 M. Abdolhosseini, S. R. Nandula, J. Song, H. Hirt and S.-U. Gorr, Peptides, 2012, 35, 231-238. 